Article

\title{
Poly[( $N$-acryloyl glycinamide)-co-( $N$-acryloyl L-alaninamide)] and Their Ability to Form Thermo-Responsive Hydrogels for Sustained Drug Delivery
}

\author{
Mahfoud Boustta and Michel Vert*(D) \\ Department of Artificial Biopolymer, Institute for Biomolecules Max Mousseron, UMR CNRS 5247, \\ Faculty of Pharmacy, University of Montpellier-CNRS-ENSCM, 15 Avenue Charles Flahault, BP 14491, \\ 34093 Montpellier CEDEX 5, France; mahfoud.boustta@umontpellier.fr \\ * Correspondence: michel.vert@umontpellier.fr; Tel.: +33-(0)411-759-723
}

Received: 1 January 2019; Accepted: 27 February 2019; Published: 3 March 2019

check for updates

\begin{abstract}
In the presence of water, poly(N-acryloyl glycinamide) homopolymers form highly swollen hydrogels that undergo fast and reversible gel $\leftrightarrow$ sol transitions on heating. According to the literature, the transition temperature depends on concentration and average molecular weight, and in the case of copolymers, composition and hydrophilic/hydrophobic character. In this article, we wish to introduce new copolymers made by free radical polymerization of mixtures of $N$-acryloyl glycinamide and of its analog optically active $N$-acryloyl L-alaninamide in various proportions. The $N$-acryloyl L-alaninamide monomer was selected in attempts to introduce hydrophobicity and chirality in addition to thermo-responsiveness of the Upper Critical Solubilization Temperature-type. The characterization of the resulting copolymers included solubility in solvents, dynamic viscosity in solution, Fourrier Transform Infrared, Nuclear Magnetic Resonance, and Circular Dichroism spectra. Gel $\rightarrow$ sol transition temperatures were determined in phosphate buffer $(\mathrm{pH}=7.4$, isotonic to $320 \mathrm{mOsm} / \mathrm{dm}^{3}$ ). The release characteristics of hydrophilic Methylene Blue and hydrophobic Risperidone entrapped in poly( $N$-acryloyl glycinamide) and in two copolymers containing 50 and $75 \%$ of alanine-based units, respectively, were compared. It was found that increasing the content in $N$-acryloyl-alaninamide-based units increased the gel $\rightarrow$ sol transition temperature, decreased the gel consistency, and increased the release rate of Risperidone, but not that of Methylene Blue, with respect to homo poly( $N$-acryloyl glycinamide). The increase observed in the case of Risperidone appeared to be related to the hydrophobicity generated by alanine residues.
\end{abstract}

Keywords: $N$-acryloyl glycinamide; $N$-acryloyl glycinamide (NAGA); $N$-acryloyl L-alaninamide; NAGA-co-N-acryloyl L-alaninamide chiral analog (NALALA) copolymers; drug delivery; gel $\leftrightarrow$ sol transition; Upper Critical Solution Temperature (UCST); thermo-responsive hydrogel

\section{Introduction}

Polymeric hydrogels are swollen 3D matrices composed of at least $50 \%$ or more of aqueous medium. Two domains of applications of hydrogels are drug delivery and tissue engineering [1-6]. In such hydrogels, water is entrapped in a 3D network of chemically or physically cross-linked macromolecules. Contrary to chemical cross-linking, physical cross-linking can be reversible under the action of a change in environmental conditions. The change is referred to as a stimulus when the action is desired. Basically, the reversibility of the physical cross-linking present in hydrogels results in gel $\leftrightarrow$ sol transition. Stimuli like salt concentration, $\mathrm{pH}$, ionic strength, and temperature changes have been proposed as a means to modify water-polymer interactions and lead to sol $\leftrightarrow$ gel reversible 
transitions [5]. Many injectable hydrogels based on artificial polymers or on biopolymers have been studied over the past decades for their interest in various domains of therapy [7,8]. In mammalians, ionic strength, $\mathrm{pH}$, and temperature are set at physiological values that may serve as references to make an in situ sol $\rightarrow$ gel transition respectful of living elements (proteins, cells, etc.) and exploitable in therapy. For instance, a stimulus-responsive gel-forming system can be injected in the sol form slightly above or below such a physiological reference, and in gel in situ soon after being under physiological conditions. With respect to this, temperature has been considered particularly attractive. Among the thermo-responsive polymer-water systems, a majority is based on the Low Critical Solution Temperature (LCST) phenomenon, i.e., they are solutions at temperatures lower than body temperature $[9,10]$. LCST-based transitions are generally due to dehydration phenomena, and thus results in water expulsion and matrix contraction. Although less frequently, thermo-responsive hydrogels based on the Upper Critical Solution Temperature (UCST) phenomenon are also present in the literature $[9,11]$. When sol is above the transition temperature, the aqueous medium turns to a gel on cooling. In this case, gelation is generally due to the formation of a network of hydrogen bonds with almost no contraction. Gelatin is the archetype of such hydrogels. However, gelatin is rapidly degraded in vivo and has a sol $\rightarrow$ gel transition temperature around $32{ }^{\circ} \mathrm{C}$ that is too low relative to body temperature.

It is now well known that some poly(N-acryloyl glycinamide) polymers (PNAGA)-water systems exhibit reversible sol $\leftrightarrow$ gel transitions of the UCST type [12]. In such systems, the polymer is soluble above the UCST, whereas more or less consistent hydrogels are rapidly formed below this [13-16]. Polymer concentration, molecular weights, and copolymerization are factors that can be exploited to modify the temperature of phase separation or gelation. $\mathrm{N}$-acryloyl glycinamide (NAGA) was copolymerized with different co-monomers, namely $N$-acetyl acrylamide, acrylic acid, butyl acrylate, styrene, biotin-functionalized methacrylamide, etc. [12,17-19]. When occurring, the UCST-dependent phase separation was affected by the presence of the co-monomers and even suppressed when carboxylic functions were present [12,18].

In a previous publication, we have shown that drug-containing PNAGA hydrogels present an interesting potential to fulfill many of the properties required for drug delivery after injection under warm sol form [20,21]. The potential was investigated using Methylene Blue as a model of hydrophilic drugs [21]. In vitro, the release of this dye was diffusion-controlled and sustained release was demonstrated in vivo. In contrast, Risperidone, a hydrophobic drug, loaded in a PNAGA hydrogel exhibited solubility-controlled zero order release [21], the release rate being slowed down when $\mathrm{Mg}(\mathrm{OH})_{2}$ was present in the formulation [22].

This article aims at exploring a new type of optically active and thermo-responsive polymer obtained by copolymerization of NAGA and its $N$-acryloyl L-alaninamide chiral analog (NALALA) (Figure 1).
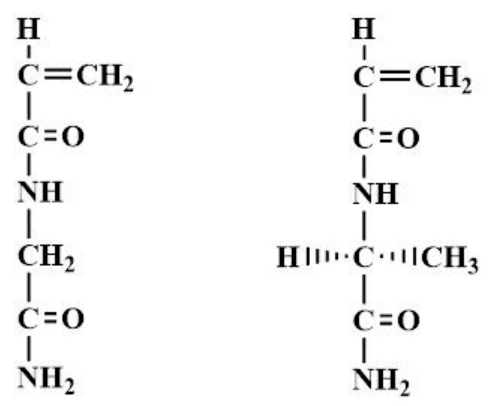

Figure 1. Chemical formula of $N$-acryloyl glycinamide (NAGA) (left) and $N$-acryloyl L-alaninamide chiral analog (NALALA) (right). 
Based on the protocol exploited for the free-radical polymerization of NAGA, copolymers combining NAGA and NALALA-derived units were synthesized using isopropanol as transfer agent to control and adjust molecular weights. The characterization of homo and copolymers made from feeds containing $0,10,25,50,75$, and 100\% NALALA included solubility in water and solvents, Fourier Transform Infrared (FTIR) and Nuclear Magnetic Resonance (NMR) spectra, dynamic viscosity, chiroptical properties, and gel $\rightarrow$ sol transition temperature. Methylene Blue and Risperidone, used previously to test the potential of PNAGA hydrogels, were individually combined with the copolymers derived from approximately 50:50 and 25:75 M:M NAGA:NALALA polymerization feeds. Release profiles were discussed comparatively. All data were used to discuss the influence of the presence of NALALA methyl groups on the properties of the copolymers relative to homopolymers.

\section{Results and Discussion}

\subsection{NAGA and NALALA Monomers}

NALALA was synthesized using a protocol similar to that reported for NAGA [20,21] modified from [13]. Both compounds were soluble in aqueous media. Figure 2 compares the ${ }^{1} \mathrm{H}-\mathrm{NMR}$ spectra of NAGA and NALALA in d6-DMSO, with respective peak assignments. The two spectra are rather comparable in the 8.5-5.5 ppm range. Below $5.5 \mathrm{ppm}$, the NALALA methyne 2 resonance (quintuplet at $4.31 \mathrm{ppm}$ with $\mathrm{J}=7.28 \mathrm{~Hz}$ ) is located downfield compared to the NAGA $\mathbf{d}$ methylene one (doublet at $3.65 \mathrm{ppm}$ ). The presence of the quintuplet shows that the protons of the $\mathrm{CH}-\mathrm{CH}_{3}$ group were coupled with the proton of the vicinal NH amide group. In the NAGA spectrum, the methylene $\mathbf{d}$ protons and the NH proton were also coupled and appears as doublet and triplet, respectively. Furthermore, the NALALA spectrum presents a doublet at $1.22 \mathrm{ppm}$ specific of methyl $\mathbf{1}(\mathrm{J}=7.11 \mathrm{~Hz})$.

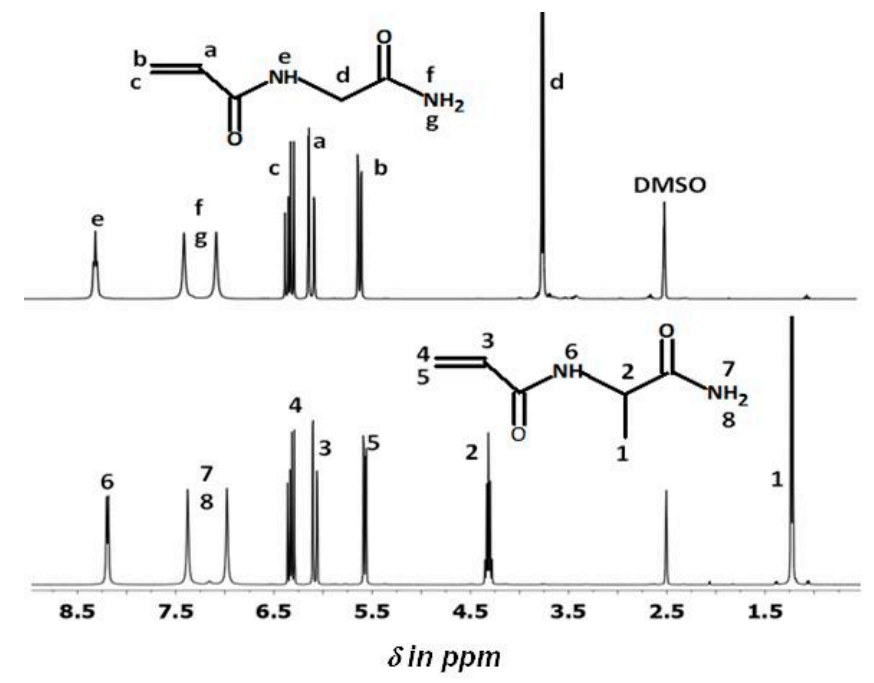

Figure 2. Comparison of the $400 \mathrm{MHz}{ }^{1} \mathrm{H}-\mathrm{NMR}$ spectra of NAGA (top) and NALALA (bottom) in d6-DMSO.

Figure 3 shows the ${ }^{13} \mathrm{C}-\mathrm{NMR}$ spectrum of NALALA in d6-DMSO with the peak assignment that agrees with the structure deduced from the ${ }^{1} \mathrm{H}-\mathrm{NMR}$ spectrum in Figure 2. 


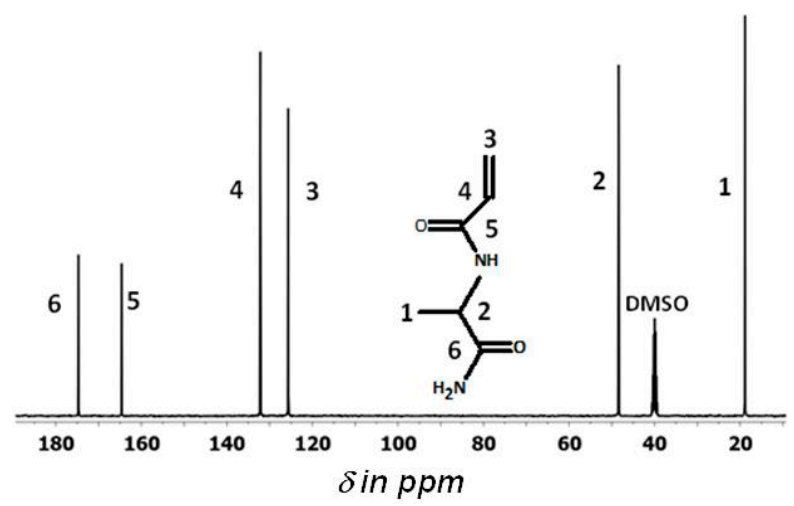

Figure 3. The ${ }^{13} \mathrm{C}-\mathrm{NMR}$ (400 MHz proton) spectrum of NALALA in d6-DMSO.

\subsection{Synthesis and Characterization of Poly(NAGA-co-NALALA) Copolymers}

It was previously reported that the molecular weight of PNAGA polymers synthesized by free radical polymerization can be controlled using isopropanol as the transfer agent [15]. The method was applied to mixtures of NAGA and NALALA and yielded PNAGA-co-NALALA copolymers irrespective of the proportion of the two acrylic monomers in the feed. Compositions in glycinamide and L-alaninamide-derived units deduced from ${ }^{1} \mathrm{H}$-NMR spectra (Figure 4) were close to those of the corresponding feeds after purification by dialysis and freeze drying (Table 1). This finding argued in favor of monomers with similar reactivity.

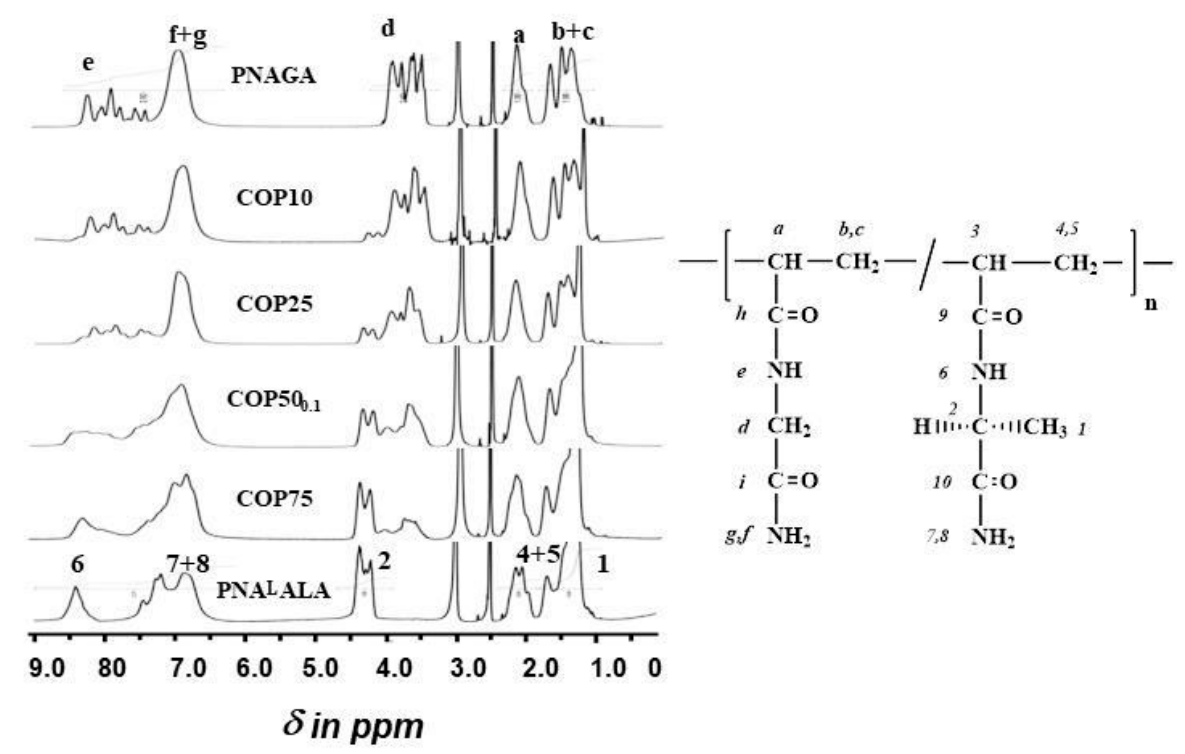

Figure 4. The ${ }^{1} \mathrm{H}-\mathrm{NMR}$ spectra of homopolymers and copolymers in d6-DMSO at $100{ }^{\circ} \mathrm{C}$ (left), and assignments of the resonances to the different protons present in NAGA units (numbers) and in NALALA ones (letters) (right).

According to the normal action of transfer agents in chain polymerization of vinylic and acrylic monomers, the higher the concentration in transfer agent, the lower the molecular weight is. Therefore, the molecular weights of the polymers were likely to increase when the transfer agent concentration decreased from 1 to $0.1 \mathrm{M}$, a trend confirmed by dynamic viscosity data given in Table 1, where abbreviations of copolymers are composed of the acronym COP followed by a number that reflects the percentage of NALALA-based units in the polymerization medium and a subscript number that reflects the concentration of isopropanol in the polymerization medium. 
Table 1. PNAGA, PNAGA-co-NALALA, and PNALALA polymers obtained by radical polymerization in water at $60^{\circ} \mathrm{C}$ initiated by $5 \times 10^{-4} \mathrm{M} \mathrm{K}_{2} \mathrm{~S}_{2} \mathrm{O}_{8}$ in the presence of different amounts of isopropanol transfer agent.

\begin{tabular}{|c|c|c|c|c|c|c|c|}
\hline \multirow{3}{*}{ Polymer } & \multirow{2}{*}{\multicolumn{2}{|c|}{$\begin{array}{c}\text { NALALA in the } \\
\text { Monomer Feed } W / W \%\end{array}$}} & \multirow{3}{*}{$\begin{array}{l}\text { Isopropanol } \\
\mathrm{mol} / \mathrm{dm}^{3}\end{array}$} & \multirow{3}{*}{$\begin{array}{c}\text { Yield } \\
\%\end{array}$} & \multicolumn{3}{|c|}{ Behavior in Saline at Room Temperature } \\
\hline & & & & & \multicolumn{2}{|c|}{ Medium Aspect ${ }^{2}$} & \multirow{2}{*}{$\begin{array}{c}\text { Dynamic Viscosity Pa.s } \\
1 \% \mathrm{~W} / \mathrm{V}^{3}\end{array}$} \\
\hline & Feed & Polymer $^{1}$ & & & $1 \% W / V^{3}$ & $5 \% W / V^{3}$ & \\
\hline $\mathrm{PNAGA}_{1}$ & 0 & 0 & 1 & 93 & 0 & 5 & $1.401^{4}$ \\
\hline $\mathrm{COP} 10_{1}$ & 10 & $9 \pm 1$ & 1 & 95 & 0 & 5 & 1.411 \\
\hline $\mathrm{COP} 25_{0.25}$ & 25 & $22 \pm 2$ & 0.25 & 93 & 0 & 6 & 1.778 \\
\hline $\mathrm{COP}_{50}$ & 50 & $53 \pm 3$ & 1 & 93 & 0 & 2 & 1.322 \\
\hline $\mathrm{COP} 5_{0.25}$ & 75 & $73 \pm 5$ & 0.25 & 96 & 0 & 4 & 1.918 \\
\hline PNALALA $_{1}$ & 100 & 100 & 1 & 90 & 1 & 3 & n.a. \\
\hline
\end{tabular}

Note: ${ }^{1}$ From ${ }^{1} \mathrm{H}-\mathrm{NMR} ;{ }^{2}$ Physical form: 0: clear solution; 1 : solution slightly turbid; 2: clear flowing soft gel; 3: turbid soft and breakable gel; 4: turbid cohesive soft gel; 5 : clear breaking gel; 6 : elastic gel; ${ }^{3}$ Concentration in polymer; ${ }^{4}$ Molecular weight of 117,000 according to the method proposed by Haas [15].

Table 1 shows that the physical aspect of the different polymers in saline at room temperature depended on the concentration of the polymerization medium in transfer agent for a given concentration in monomers $\left(\mathrm{mol} / \mathrm{dm}^{3}\right.$ ) (comparison of COP50 $0.1,0.25$ and 1 ) and on the composition in monomers (comparison of COP25 $5_{0.25}, \mathrm{COP} 50_{0.25}$, and $\mathrm{COP} 75_{0.25}$ ) in the aqueous polymerization medium. Gel formation depended also on the polymer concentration. At $1 \% \mathrm{~W} / \mathrm{V}$, none of the copolymers gelled at room temperature except $\mathrm{COP} 50_{0.1}$, which had the higher molecular weight. At $5 \%$, all the polymers were under gel form. However, the gels were more or less consistent and cohesive, as shown by the ranking from grade 3 (turbid soft gel) to grade 6 (elastic gel). Therefore, it was concluded that the lower the transfer agent concentration, the higher the molecular weight and the higher the consistency of the gel formed.

The PNALALA homopolymer formed gels regardless of the polymer concentration but they were fragile and breakable. The lack of suitable organic solvent, the need of a H-bond breaking salt or of high temperature in water, and the presence of residual interactions between macromolecules in solutions appeared technical obstacles to using Size Exclusion Chromatography (SEC) for molecular weight determination. Intrinsic viscosity was also problematic because of intermolecular interactions and concentration-dependent gelation. Although dynamic viscosity was not appropriate to measure molecular weights, this technique was selected as a compromise to compare the different copolymers. Data in Table 1 shows a remarkable correlation between the decrease of the concentration in transfer agent and the increase of dynamic viscosity, since $1,0.25$, and $0.1 \mathrm{M}$ in transfer agent corresponded to approximately 1.4, 1.8, and $8 \mathrm{~Pa} \cdot \mathrm{s}$, respectively. Based on the molecular weight of the PNAGA 1 homopolymer $(117,000 \mathrm{~g} / \mathrm{mol})$, evaluated by viscometry according to the method proposed by Haas [15], a dynamic viscosity of approximately 1.4 Pa.s likely corresponded to molecular weights in the range of $110,000-130,000 \mathrm{~g} / \mathrm{mol}$.

\subsection{Fourrier Transform Infrared}

The spectra of the different polymers appeared to be composed of broad absorption zones that included enlarged and overlapping bands, as is usual for acrylic polymers synthesized by free radical polymerization. The spectra looked similar and could hardly be differentiated by this technique, especially when the only structural difference is a proton replaced by a methyl group. In agreement with the structure of the homo and copolymers, the broad zone located between 3650 and $2900 \mathrm{~cm}^{-1}$ included free and bonded $\mathrm{NH}$ and $\mathrm{NH}_{2}$ stretching vibrations above $3000 \mathrm{~cm}^{-1}$, and three small peaks on the $3000-2900 \mathrm{~cm}^{-1}$ shoulder due to $\mathrm{CH}, \mathrm{CH}_{2}$, and $\mathrm{CH}_{3}$ stretching. Another group of bands was found in the $1690-1500 \mathrm{~cm}^{-1}$ region composed of overlapping primary and secondary amide I and II bands followed by $\mathrm{CH}, \mathrm{CH}_{2}$, and $\mathrm{CH}_{3}$ bending deformation bands around $1450 \mathrm{~cm}^{-1}$. 


\subsection{Nuclear Magnetic Resonance}

Figure 4 shows the ${ }^{1} \mathrm{H}-\mathrm{NMR}$ spectra of the different copolymers in comparison with corresponding homopolymers. The peaks were assigned according to the formula (Figure 4 right) using the spectra of the homopolymers as references. The composition of the copolymers in NALALA units (Table 1) was calculated using the ratio $2 \times$ (methyne 2 )/(methylene $\mathbf{d}$ ) resonances areas corresponding to NAGA and NALALA units, respectively.

Figure 5 shows the variation of the composition of copolymers relative to the composition of monomer feeds. The linearity confirmed the comparable reactivity of the two monomers.

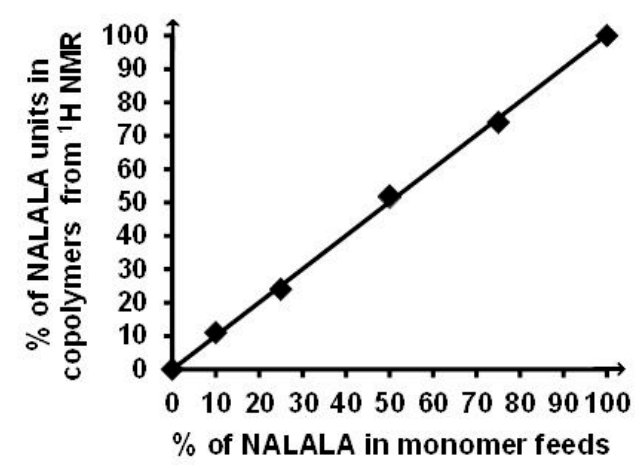

Figure 5. Linear correlation between copolymer and feed compositions. Differences between replicates are inside the size of data points.

Figure 6 shows the ${ }^{13} \mathrm{C}-\mathrm{NMR}$ spectra of the copolymers in comparison with those of the homopolymers. The presence of NALALA-based units is clearly observed at the level of $48 \mathrm{ppm}$ (carbon 2) and at 18.2 ppm (carbon $\mathbf{1}$ ).

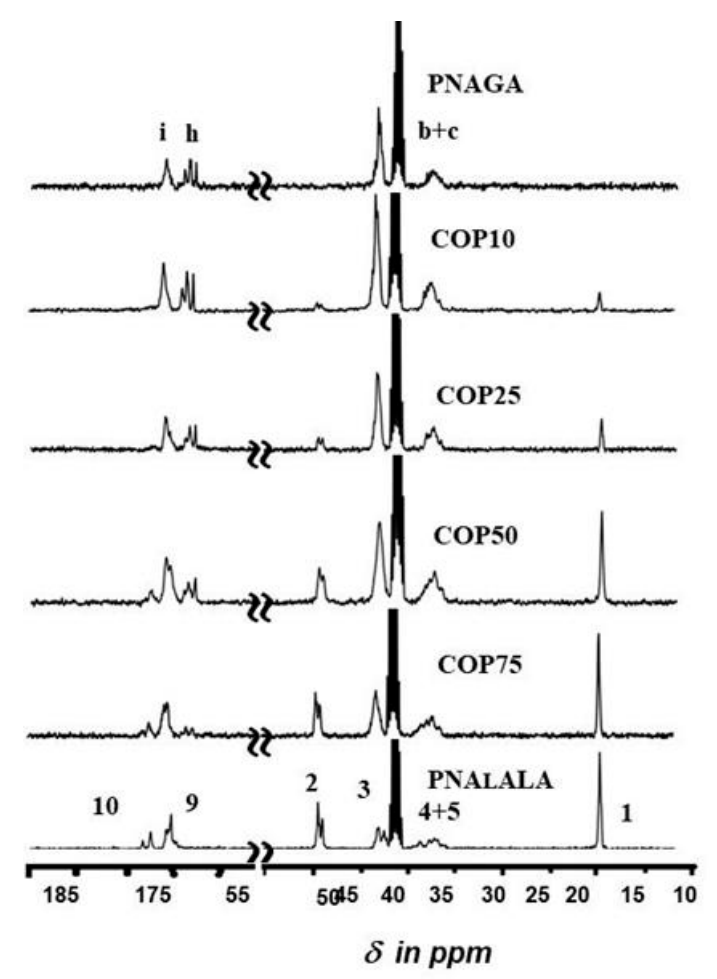

Figure 6. The ${ }^{13} \mathrm{C}-\mathrm{NMR}$ spectra (bottom) of homopolymers and copolymers in DMSO at $100{ }^{\circ} \mathrm{C}$. Assignments of the various carbon atom resonances are based on the formula in Figure 4 (right). 


\subsection{Circular Dichroism}

Figure 7 left shows the CD spectra of PNALALA at two concentrations in water. As for any unordered aliphatic polyamide chain, the profile resulted from the overlapping of the $n-\sigma^{*}$ and the $\pi-\pi^{*}$ electronic transitions [23]. The copolymers with NAGA exhibited similar profiles that were directly proportional to the content in NALALA units as shown in Figure 5 right.
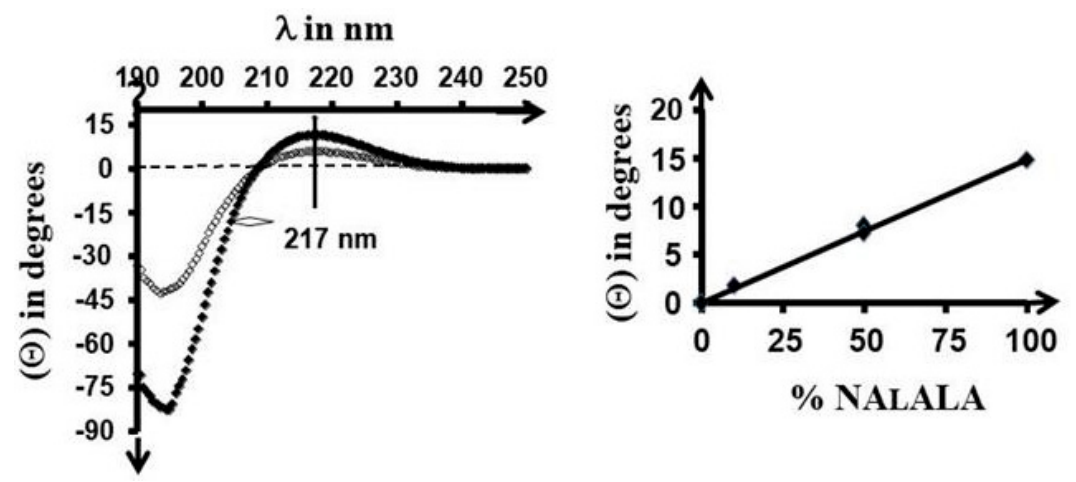

Figure 7. Circular Dichoïsm (CD) spectra of PNALALA ם: [C] $=0.25 \% \mathrm{~W} / \mathrm{V} ; \square:[C]=0.125 \% w / v$ in water at $20^{\circ} \mathrm{C}$ (left) and changes of ellipticity $(\theta)$ at $217 \mathrm{~nm}$ (right) as a function of the composition of copolymers in NALALA-based units deduced from the ${ }^{1} \mathrm{H}-\mathrm{NMR}$ spectra shown in Figure 3. Differences in ellipticity of replicates are inside the size of data points, as shown by duplicates for $50 \%$ NALALA.

This finding shows that NAGA units did not perturb the chiral properties due to NALALA ones. Therefore, ellipticity was used to assess the composition of copolymers, as it was performed from NMR data. The linearity agreed with the previous conclusion relative to comparable reactivity of the two monomers.

\subsection{Reversible Gel $\leftrightarrow$ Sol Transitions}

PNAGA-co-NALALA copolymers with similar composition and different molar sizes according to transfer agent and dynamic viscosity $\left(\mathrm{COP} 50_{0.1,0.25}\right.$, and 1$)$ or similar molar size and different compositions (COP25 $5_{0.25}, 50_{0.25}$, and $75_{0.25}$ ) were selected to investigate the influence of these characteristics on the concentration-dependence of the gel $\rightarrow$ sol transition (Figure 8). From the various profiles, it was concluded that the three factors were interdependent. The higher the concentration or the higher the content in alanine-based units (comparison of COP25 $5_{0.25}, 50_{0.25}$, and $75_{0.25}$ ), the higher the transition temperature was, and the higher the concentration and the higher the molecular weight (comparison of $\mathrm{COP} 50_{0.1}, 0.25$, and 1 ), the higher the transition temperature was.

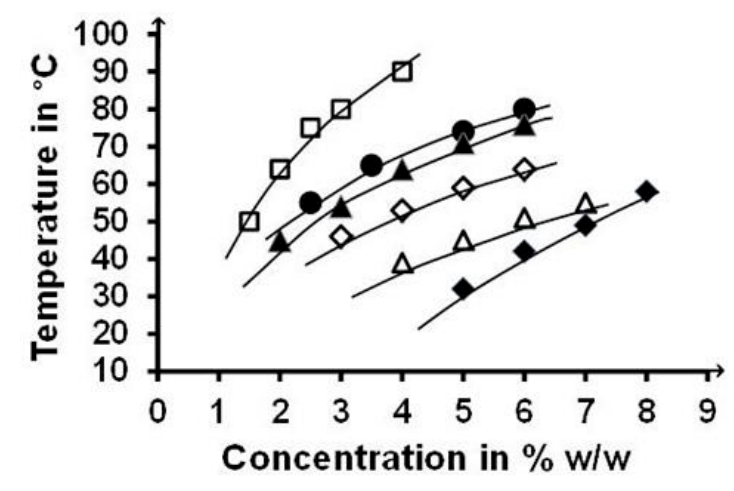

Figure 8. Gel $\rightarrow$ sol transition temperatures of PNAGA-co-NALALA of different compositions synthesized using different concentrations of transfer agent in the feed: $\square$ Cop75 $0.25 ; \bullet C_{0} 50_{0.1} ; \boldsymbol{\Delta}$ Cop $50_{0.25} ; \bullet$ Cop50 1 ; $\diamond$ Cop25 $5_{0.25} ; \Delta$ Cop10 . Differences between replicates are inside the size of data points. 


\subsection{Release Sustaining}

The potential of NALALA-based polymers relative to drug delivery was estimated using Methylene Blue and Risperidone as models of hydrophilic and hydrophobic drugs, respectively, as performed in the case of a PNAGA homopolymer [21,24]. Figure 9 compares the release profiles observed for Methylene Blue loaded in gels made of a PNAGA homopolymer and a copolymer rich in NALALA-based units synthesized in the presence of the same concentration in transfer agent, and thus considered as comparable in terms of molecular weight. There was no significant difference between the two release profiles, thus showing that the presence of NALALA-based units did not affect the diffusion-controlled release typical of PNAGA.

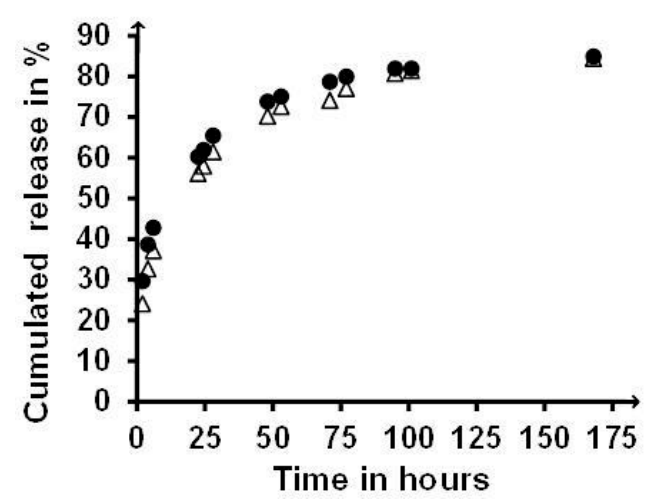

Figure 9. Comparison of the cumulated release profiles of the methylene released from $\mathrm{COP} 50_{0.1}$ $(\Delta)$ and PNAGA $0.1(\bullet)$ (gels from $6 \%$ polymer solutions) at $37^{\circ} \mathrm{C}$ as determined by Ultraviolet (UV) spectroscopy at $665 \mathrm{~nm}$ (see the experimental section).

Similarity between release profiles was also observed in the case of the hydrophobic Risperidone in PNAGA and COP50 0.1 gels for two polymer concentrations, as shown in Figure 10. In contrast, a significant increase of release rate was observed for the COP75 0.25 copolymer containing a majority of NALALA-based units, with the initial burst staying rather low $(<10 \%)$. In all cases, linear release profiles typical of solubility-control were observed. The rate increase observed in the case of the COP75 was tentatively assigned to the effect of the extra methyl groups present in NALALA-based units on hydrophobicity-related polymer-water interactions that condition the formation of hydrophobic microdomains, where hydrophobic molecules, including drugs, can be accommodated [24-26]. In practice, such accommodation corresponds to an increase of dispersed molecules more prone to diffuse in the external aqueous medium than if they were aggregated in dispersed solid particles. The enhancing effect of NALALA-based units present in the COP75 0.25 copolymer should have been larger for the PNALALA homopolymer. However, the poor characteristics of the gel did not allow obtainment of significant release profiles because of weakness related to hydrophobic interactions. This correlation was supported by the fact that a dried PNALALA gel was very difficult to solubilize again due to the establishment of short-distance macromolecule-macromolecule hydrophobic interactions on top of the amide-related hydrogen bonds. Such short-distance interactions were less energetic in swollen gels but still effective enough to significantly increase the transition temperatures, as shown by the location of the COP75 0.25 profile in the temperature scale (Figure 8 ). Sol $\leftrightarrow$ gel transition temperatures high above body temperature were regarded as a possible drawback relative to using thermos-responsive injectable hydrogels in the field of drug delivery. 


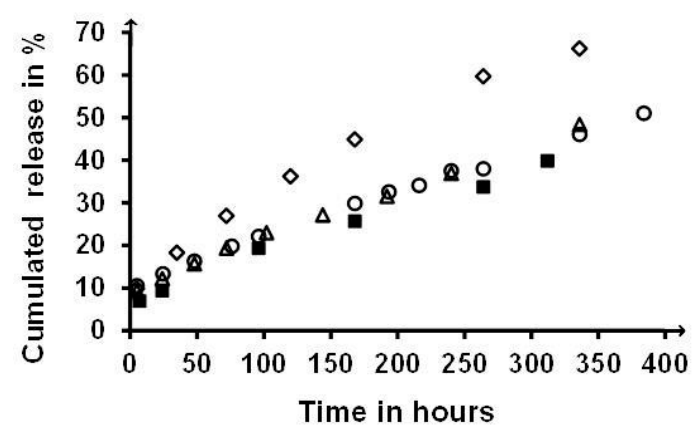

Figure 10. Comparison of Risperidone release profiles from different hydrogels $\left(1 \mathrm{~cm}^{3}\right.$ containing $30 \mathrm{mg}$ of Risperidone) in gently stirred $600 \mathrm{~cm}^{3}$ of iso-osmolar phosphate buffer at $\mathrm{pH}=7.4$ and $37^{\circ} \mathrm{C}$ : 口: $\mathrm{PNAGA}_{1}$ at $6 \% \mathrm{~W} / \mathrm{V} ; \triangle \mathrm{COP} 50_{0.1}$ at $6 \% \mathrm{~W} / \mathrm{V} ; \bigcirc \mathrm{COP}_{0.1}$ at $5 \% \mathrm{~W} / \mathrm{V}$, and $\diamond \mathrm{COP}_{0.25}$ at $5 \%$ $\mathrm{W} / \mathrm{V}$. Profiles were averaged from duplicates, and from triplicate for the COP75 0.25 . Differences were all smaller than the data point symbols.

\section{Conclusions}

This work shows that thermo-responsive PNAGA-co-NALALA copolymers can be synthesized by radical polymerization in aqueous medium, as was the case for PNAGA homopolymers. At $5 \%$ $w / v$ and slightly above, all the investigated copolymers exhibited UCST-type gelation with molecular weight and concentration-dependences of the gel $\leftrightarrow$ sol transition. The gels were more or less cohesive and consistent depending on the composition that was accessible from the polymerization feed, and from NMR and CD spectra. Some of the gels showed a potential to serve as injectable drug delivery systems under the sol form below $50{ }^{\circ} \mathrm{C}$, as in the case of PNAGAs. The release of the Methylene Blue hydrophilic model was diffusion-controlled, whereas that of Risperidone was linear and thus solubility-controlled. The greater release rate observed for the copolymer rich in NALALA-base units was tentatively assigned to the associated hydrophobicity known to increase the local solubility of hydrophobic compounds by dissolution in lipophilic microdomains, as observed for many other amphiphilic copolymers. Unfortunately the effect could not be amplified using the PNALALA homopolymer. The exploitation of COP copolymers rich in NALALA-base units as injectable hydrogels seems possible provided the sol $\rightarrow$ gel transition temperatures allow injection under tissue-respecting conditions, as was possible in the case of some PNAGAs.

\section{Materials and Methods}

\subsection{Chemicals}

The L-Alaninamide hydrochloride was purchased from Ark Pharm (Libertyville, IL, USA). Acryloyl chloride, potassium carbonate, 2-propanol, acetone, methanol, diethyl ether, and dichloromethane were supplied by Aldrich (Saint-Quentin, Fallavier, France). Glycinamide hydrochloride was obtained from Acros Organics Fisher Scientific (Illkirch, France). Di-potassium peroxodisulfate was used as received from VWR (Fontenay-sous-Bois, France). The isoosmolar $\mathrm{pH}=7.4 / 320 \mathrm{mOsm}$ phosphate buffer was made by mixing $3.31 \mathrm{~g}$ of $\mathrm{NaH}_{2} \mathrm{PO}_{4} \cdot \mathrm{H}_{2} \mathrm{O}$ and $33.77 \mathrm{~g}$ of $\mathrm{Na}_{2} \mathrm{HPO}_{4} \cdot 7 \mathrm{H}_{2} \mathrm{O}$ in $1 \mathrm{dm}^{3}$ of water. Spectra/Por ${ }^{\circledR}$ membranes were supplied by Roth Sochiel (Lauterbourg, France). The $\mathrm{N}$-acryloyl glycinamide was synthesized as reported in our previous study [21].

\subsubsection{Synthesis of N-Acryloyl L-Alaninamide (NALALA)}

The L-alaninamide hydrochloride ( $25 \mathrm{~g}, 0.2 \mathrm{~mol}$ ) was introduced in a $1 \mathrm{dm}^{3}$ three-necked reactor equipped with a mechanical stirrer and placed in an ice bath. Argon was allowed to gently flow through the reactor. Next, $50 \mathrm{~cm}^{3}$ of cool water was added and the mixture was stirred until complete dissolution. Then, $300 \mathrm{~cm}^{3}$ of cool diethyl ether and $140 \mathrm{~cm}^{3}$ of $\mathrm{cool} 2 \mathrm{M}\left(\mathrm{mol} / \mathrm{dm}^{3}\right.$ potassium carbonate were introduced successively, prior to dropwise addition of a solution of $20 \mathrm{~g}(0.22 \mathrm{~mol})$ of freshly 
distilled acryloyl chloride in $100 \mathrm{~cm}^{3}$ of diethyl ether. The mixture was stirred at $0{ }^{\circ} \mathrm{C}$ during the addition. At the end, stirring was maintained for $1 \mathrm{~h}$ at room temperature. The $\mathrm{pH}$ of the medium was lowered to 2 by addition of $6 \mathrm{~N}\left(\left[\mathrm{H}^{+}\right] / \mathrm{dm}^{3}\right) \mathrm{HCl}$. The organic layer was removed and the aqueous medium was washed three times with $200 \mathrm{~cm}^{3}$ of diethylether. The aqueous solution was discolored with charcoal and filtered on Celite ${ }^{\circledR}$. The remaining trace of diethylether was eliminated under dynamic vacuum at $20^{\circ} \mathrm{C}$ and the $\mathrm{pH}$ was returned to neutral with $2 \mathrm{~N}\left(\left[\mathrm{OH}^{-} / \mathrm{dm}^{3}\right] \mathrm{NaOH}\right.$. Finally the mixture was freeze-dried to yield $66 \mathrm{~g}$ of a mixture of NALALA and potassium chloride. The mixture was washed three times with $400 \mathrm{~cm}^{3}$ of a $4 / 1 \mathrm{v} / \mathrm{v}$ ethanol: methanol mixture to extract the organic component. The solution was evaporated to yield $28 \mathrm{~g}$ of crude NALALA. After recrystallization in $350 \mathrm{~cm}^{3}$ of a $4 / 1 \mathrm{v} / \mathrm{v}$ ethanol/acetonitrile mixture, pure NALALA $(17 \mathrm{~g})$ was recovered. This monomer melted at $147.5{ }^{\circ} \mathrm{C}$ (yield $62 \%$ with respect to the initial amount of alaninamide).

\subsubsection{Synthesis of Poly(NAGA-co-NALALA) Copolymers}

Typically, a suitable amount of isopropanol was introduced in a $250 \mathrm{~cm}^{3}$ flask equipped with a magnetic stirrer together with suitable amount(s) of monomer(s) $\left(0.5 \mathrm{~mol} / \mathrm{dm}^{3}\right.$ in total $)$ and $10.6 \mathrm{mg}$ of potassium persulfate dissolved in $60 \mathrm{~cm}^{3}$ of deionized water. The mixture was degassed using bubbling of argon for $1 \mathrm{~h}$. The flask was then placed in an oil bath at $60^{\circ} \mathrm{C}$ and the content was allowed to polymerize for about $48 \mathrm{~h}$. At the end, the viscous mixture was diluted with $100 \mathrm{~cm}^{3}$ of hot water to obtain a diluted solution at $50{ }^{\circ} \mathrm{C}$ and introduced in a dialysis tube (6/8000 Dalton cut-off) maintained at the same temperature for $48 \mathrm{~h}$, with outer water changed several times up to the absence of diffused material. At the end, the content of the tube was recovered and freeze-dried to yield the polymer.

\subsection{Methods}

\subsubsection{Spectral Characterizations}

ATR-FTIR spectra were recorded using a FTIR spectrometer Perkin-Elmer Spectrum 100 (Villebon-sur-Yvette, France). Polymers were ground and the powder was placed on the ATR plate. The ${ }^{1} \mathrm{H}-\mathrm{NMR}$ and ${ }^{13} \mathrm{C}-\mathrm{NMR}$ spectra were recorded using a Bruker Avance III HD (Wissembourg, France) - $400 \mathrm{MHz}$ with temperature set at $100{ }^{\circ} \mathrm{C}$. Typically, approximately $30 \mathrm{mg}$ of polymer was solubilized in $0.6 \mathrm{~mL}$ of hot d6-DMSO.

\subsubsection{Dynamic Viscosity}

Dynamic viscosity data were obtained using a 4100M DMA Densimeter ${ }^{\mathrm{TM}}$ coupled with a Lovis 2000 ME microviscometer accessory from Anton Paar (Les Ulis, France). The polymers were dissolved in hot saline at a concentration of $10 \mathrm{~g} / \mathrm{dm}^{3}$ and measurements were performed at $20^{\circ} \mathrm{C}$.

\subsubsection{Thermal Characterizations}

The gel $\rightarrow$ sol transition temperature of the various polymer-water systems was determined according to the reverse test tube method. Typically, a suitable amount of dried polymer was introduced in a $4 \mathrm{~cm}^{3}$ glass tube together with deionized water or saline to make $1 \mathrm{~cm}^{3}$ of mixture that was placed in a water bath at $80^{\circ} \mathrm{C}$ up to total dissolution, generally observed after approximately $2 \mathrm{~min}$. The solution was then cooled down to room temperature to check gel formation. The gel was turned back to solution by heating at $80{ }^{\circ} \mathrm{C}$ for $5 \mathrm{~min}$ and cooled down to $0^{\circ} \mathrm{C}$ at $40{ }^{\circ} \mathrm{C} / \mathrm{min}$, left at $0{ }^{\circ} \mathrm{C}$ for $30 \mathrm{~min}$, and then heated slowly up to $80^{\circ} \mathrm{C}$ at $4{ }^{\circ} \mathrm{C} / \mathrm{min}$. A first gel $\rightarrow$ sol transition temperature was determined when the gel started to flow along the tube wall. This cycle was repeated 3 times to yield four successive gel $\rightarrow$ sol temperatures. $\mathrm{A} \pm 2{ }^{\circ} \mathrm{C}$ reproducibility of the transition temperature was generally observed after the second cycle. For all the hot polymer solutions forming gels on cooling toward room temperature, the sol $\rightarrow$ gel transition temperatures were systematically lower but did not differ by more than $3-4{ }^{\circ} \mathrm{C}$ from corresponding gel $\rightarrow$ sol ones. 


\subsubsection{Chiroptical Characterization}

The LALA-related optical activity of homo PNALALA and of the copolymers was determined by circular dichroism (CD) using a Jasco J810 spectrograph (Lisses, France) equipped with an air cooled $150 \mathrm{~W}$ Xenon source. Measurements were performed at $20^{\circ} \mathrm{C}$ under the following conditions $\left(C_{\text {polymer }}=0.025\right.$ and $0.0125 \mathrm{~g} / \mathrm{dm}^{3}, 0.1 \mathrm{~mm}$ cell path-length). The $\mathrm{CD}$ apparatus was purged with nitrogen to remove oxygen and ozone. The cell temperature was controlled by Peltier effect. Data were expressed in term of measured ellipticity.

\subsubsection{Formulations of Copolymer/Model Drug Hydrogels}

Typically, $66 \mathrm{mg}$ of dried copolymer was introduced in a $4 \mathrm{~cm}^{3}$ flat-bottom test tube and dissolved in $1 \mathrm{~cm}^{3}$ of phosphate buffer heated at approximately $80{ }^{\circ} \mathrm{C}$ using a water-bath. About $30 \mathrm{mg}$ of Risperidone $\left(7.3 \times 10^{-5} \mathrm{~mol}\right)$ or $1.25 \mathrm{mg}$ of methylene blue $\left(4 \times 10^{-6} \mathrm{~mol}\right)$ was then added to the hot solution and the tube containing the mixture was dipped in an iced-bath for fast gelation, a process necessary to keep the sparingly soluble Risperidone as homogeneous dispersion.

\subsubsection{Release Profiles}

Typically, a tube containing the formulation of interest was placed in a $1 \mathrm{dm}^{3}$ plastic bottle containing $600 \mathrm{~cm}^{3}$ of receiving medium. The container was attached to the oscillating plateau of a Heidolph 1000 incubator thermostated at $37^{\circ} \mathrm{C}$. Slow stirring was maintained during the whole release duration. At each selected time point, $1 \mathrm{~cm}^{3}$ aliquot was withdrawn that was not replaced by fresh medium, the volume of the receiving medium being large enough to maintain pseudo sink conditions. All the sols kept their volume after gelation, except for the 25/75 gel. The initial volume of this gel showed slow contraction during data collection. When the gel lost adherence to the glass tube, data collection was stopped. All data were expressed as mean values from duplicated or triplicated experiments.

Author Contributions: Conceptualization, M.V. and M.B.; methodology, M.V and M.B..; formal analysis, M.B.; investigation, M.B.; resources, M.V.; data curation, M.B. and M.V.; writing-original draft preparation, M.V.; writing-review and editing, M.V. and M.B.; supervision, M.V.

Funding: This research received no external funding on top of university and National Center for Scientific Research (CNRS) non-specific annual supports.

Acknowledgments: Authors are indebted to CNRS and the University Montpellier for financial support.

Conflicts of Interest: The authors declare no conflict of interest

\section{References}

1. Hoffman, A.S. Hydrogels for biomedical applications. Adv. Drug Deliv. Rev. 2002, 54, 3-12. [CrossRef]

2. Van Vlierberghe, S.; Dubruel, P.; Schacht, E. Biopolymer-Based hydrogels as scaffolds for tissue engineering applications: A review. Biomacromolecules 2011, 12, 1387-1408. [CrossRef] [PubMed]

3. Vashist, A.; Vashist, A.; Gupta, Y.K.; Ahmad, S. Recent advances in hydrogel based drug delivery systems for the human body. J. Mater. Chem. B 2014, 2, 147-166. [CrossRef]

4. Amin, S.; Rajabnezhad, S.; Kohli, K. Hydrogels as potential drug delivery systems. Sci. Res. Essay 2009, 3 , 1175-1183.

5. Qiu, Y.; Park, K. Environment-sensitive hydrogels for drug delivery. Adv. Drug Deliv. Rev. 2001, 53, 321-339. [CrossRef]

6. Macaya, D.; Spector, M. Injectable hydrogel material for spinal cord regeneration: A review. Biomed. Mater. 2012, 7, 012001. [CrossRef] [PubMed]

7. Huynh, C.T.; Nguyen, M.K.; Lee, D.S. Injectable block copolymer hydrogels: Achievements and future challenges for biomedical applications. Macromolecules 2011, 44, 6629-6636. [CrossRef]

8. Chaterji, S.; Kwon, I.K.; Park, K. Smart polymeric gels: Redefining the limits of biomedical devices. Prog. Polym. Sci. 2007, 32, 1083-1122. [CrossRef] [PubMed] 
9. Taylor, M.J.; Tomlins, P.; Sahota, T.S. Thermo-responsive Gels. Gels 2017, 3, 4. [CrossRef]

10. Liang, Y.; Qiao, Y.; Guo, S.; Wang, L.; Xie, C.; Zhai, Y.; Deng, L.; Dong, A. Thermoreversible gelation of poly(ethylene glycol)/poly(ester anhydride) triblock copolymer nanoparticles for injectable drug delivery systems. Soft Matter 2010, 6, 1915-1922. [CrossRef]

11. Shimada, N.; Kidoaki, S.; Maruyama, A. Smart hydrogels exhibiting UCST-type volume changes under physiologically relevant conditions. RSC Adv. 2014, 4, 52346-52348. [CrossRef]

12. Xu, Z.; Liu, W. Poly(N-acryloyl glycinamide): A fascinating polymer that exhibits a range of properties from UCST to high-strength hydrogels. Chem. Commun. 2018, 54, 10540-10553. [CrossRef] [PubMed]

13. Haas, H.C.; Schuler, N.W. Thermally reversible homopolymer gel systems. J. Polym. Sci. B 1964, 2, 1095-1096. [CrossRef]

14. Haas, H.C.; Moreau, R.D.; Schuler, D.W. Synthetic thermally reversible gel systems. II. J. Polym. Sci. Polym. Phys. Ed. 1967, 5, 915-927. [CrossRef]

15. Haas, H.C.; Chiklis, C.K.; Moreau, R.D. Synthetic thermally reversible gel systems. III. J. Polym. Sci. Part A Polym. Chem. 1970, 8, 1131-1145. [CrossRef]

16. Seuring, J.; Bayer, F.M.; Huber, K.; Agarwal, S. Upper Critical Solution Temperature of Poly( $N$-acryloyl glycinamide) in water: A Concealed Property. Macromolecules 2012, 45, 374-384. [CrossRef]

17. Glatzel, S.; Badi, N.; Pach, M.; Laschewsky, A.; Lutz, J.-F. Well-defined synthetic polymers with a protein-like gelation behavior in water. Chem. Commun. 2010, 46, 4517-4519. [CrossRef] [PubMed]

18. Seuring, J.; Agarwal, S. Polymers with Upper Critical Solution Temperature in Aqueous Solution. Macromol. Rapid Commun. 2012, 33, 1898-1920. [CrossRef] [PubMed]

19. Glatzel, S.; Laschewsky, A.; Lutz, J.-F. Well-Defined Uncharged Polymers with a Sharp UCST in Water and in Physiological Milieu. Macromolecules 2011, 44, 413-415. [CrossRef]

20. Boustta, M.; Colombo, P.-J.; Vert, M. Combination of Poly(N-Acryloyl Glycinamide) with at Least One Active Principle. WO 2013128373, 6 September 2013.

21. Boustta, M.; Colombo, P.-E.; Lenglet, S.; Poujol, S.; Vert, M. Versatile UCST-based thermoresponsive hydrogels for loco-regional sustained drug delivery. J. Control. Release 2014, 174, 1-6. [CrossRef] [PubMed]

22. Boustta, M.; Vert, M. A method to slow down the ionizationdependent release of risperidone loaded in a thermoresponsive poly(N-acryloylglycinamide) hydrogel. Drug Deliv. Transl. Res. 2017, 7, 460-464. [CrossRef] [PubMed]

23. Braud, C.; Vert, M. Chiroptical properties of optically active (-) poly N-sec-butyl-N-methyl acrylamide] with regard to amide chromophores. Isr. J. Chem. 1976, 15, 39-45. [CrossRef]

24. Braud, C.; Vert, M. Poly ( $\beta$-malic acid) as a source of polyvalent drug carriers: Possible effects of hydrophobic substituents in aqueous media. In Polymers as Biomaterials; Shalaby, S.W., Hoffman, A.S., Ratner, B.D., Horbett, T.A., Eds.; Plenum Publ. Co.: New York, NY, USA, 1984.

25. Vert, M. Polyvalent polymeric drug carriers. In CRC Critical Reviews-Therapeutic Drug Carrier Systems; Bruck, S.D., Ed.; CRC Press: Boca Raton, FL, USA, 1986; pp. 291-327.

26. Domurado, D.; Vert, M. Bioresorbable polyelectrolyte amphiphiles as nanosized carriers for lipophilic drug solubilization and delivery. J. Biomater. Sci. Polym. Ed. 2007, 18, 287-301. [CrossRef] [PubMed]

(C) 2019 by the authors. Licensee MDPI, Basel, Switzerland. This article is an open access article distributed under the terms and conditions of the Creative Commons Attribution (CC BY) license (http:// creativecommons.org/licenses/by/4.0/). 